Domenico Cernecca
Zagreb

\title{
CONTATTO LINGUISTICO E TRADUZIONE
}

1. Basta dare un'occhiata alle vetrine di una buona libreria per farsi un'idea dell'importanza che la traduzione riveste nella vita intellettuale del mondo moderno. Infatti, oltre che essere lo speccio della cultura nazionale, ogni libreria, con la molteplicità dei nomi stranieri e i titoli dei libri tradotti, è la testimonianza più viva di quel cosmopolitismo che permea ormai le più larghe zone del tessuto culturale della famiglia umana.

Ma se il numero delle produzioni e degli autori che hanno l'onore della stampa e arrivano in libreria è grande, immensamente più grande ancora è la folla degli utenti dell'istituto della traduzione, se si tien conto che la società moderna è giunta a un tal grado di integrazione culturale, che quotidianamente, sotto le forme più diverse e per $\mathrm{i}$ motivi più disparati, tutti gli strati della popolazione giungono a contatto coi prodotti linguistici stranieri [parole, frasi o passi interi] da interpretare e capire. In un certo senso traducono, anche senza averne chiara coscienza, tutti: l'utentè di una macchina straniera recante indicazioni sull'uso nella rispettiva lingua, il commerciante che offre ai clienti un prodotto esotico, il giovanotto che fischietta per la strada l'ultima canzone di successo di uno dei tanti festivals internazionali. Non sarà perciò esagerato affermare che l'attività interpretativa della parola straniera è uno dei fenomeni più diffusi e una caratteristica del mondo moderno, anche se il tradurre è un mestiere vecchio quanto il mondo stesso, spingendo le sue radici, esili e filiformi quanto si vuole, fino alle prime comunità umane che stabilirono fra loro un qualunque contatto. Non per niente la coscienza della pluralità dei linguaggi simboleggiata nella torre di Babele è una delle nozioni più antiche implicante un sempre rinnovato sforzo di comprensione, particolarmente fertile e proficuo per il comune progresso del genere umano.

Se la scienza della lingua ha individuato nelle zone di frontiera le radici del contatto linguistico e del conseguente bilinguismo, oggi si può costatare che questo fronte si è articolato ed esteso fino ad incidere più o meno profondamente nel tessuto nazionale di ogni paese civile seguendo le linee di un processo storico particolare, per cui le civiltà che si sus- 
seguirono dalla notte dei tempi non sarebbero state possibili o non avrebbero assunto la forma che ebbero senza l'istituto della traduzione. Non si può infatti comprendere la civiltà latina senza quella dei greci, né sarebbe mai sorta la civiltà del Rinascimento, che dai greci, dai romani e dagli arabi trasse gl'impulsi fecondi, se fosse mancata l'opera dei traduttori, questi costruttori di ponti ideali fra lingue e culture diverse. Quanti furono i traduttori disseminati su quest'arco di secoli di contatti umani? Quelli i cui nomi ci sono stati tramandati non sono molti, ma eccellenti, se si pensa a un Cicerone bilingue latino-greco, a S. Gerolamo, traduttore della Vulgata, ad Avicenna, a Lutero che con la traduzione della Bibbia gettò le basi del tedesco moderno.

Al problema della comunicazione interlinguistica non doveva e non poteva rimaner sorda, prima di tutti, la scuola, la quale avvertì fin dagli inizi la necessità di organizzare lo studio delle lingue straniere di prestigio culturale, ed è per questo che nella scuola romana incontriamo lo studio della lingua greca. Diminuiti o resi difficili i contatti linguistici delle comunità chiuse del feudalesimo, la chiesa mantiene e diffonde fra tutti l'uso della lingua latina che soddisfa le esigenze del culto ed è il veicolo internazionale della filosofia, della scienza, della cultura e dell'attività diplomatica.

Con l'affacciarsi della borghesia alla ribalta della storia e con la conseguente formazione delle culture e delle lingue nazionali, l'espansione commerciale e militare si accompagna allo studio delle lingue dei paesi vicini e lontani, e specialmente del francese, che nel XVIII secolo diventa il principale veicolo della civiltà occidentale, mentre nel XIX prende il sopravvento il tedesco e poi l'inglese, ${ }^{1}$ la cui importanza quale lingua dei contatti internazionali a tutti i livelli cresce specialmente dopo la seconda guerra mondiale.

In tutto questo travaglio è la scuola che, con la sua esperienza pedagogica e didattica, in uno sforzo organizzato e continuo, rende sempre più facile, più rapido ed efficace l'apprendimento delle lingue vive. Essa svolge una silenziosa, ma costante opera di valutazione e di selezione scartando lentamente il latino e creando metodi e strumenti didattici sempre più efficaci e precisi. Partendo delle forme classiche di studio indirette, fondate sulla conoscenza grammaticale e sulla traduzione del testo scritto, si giunge alle varie forme assunte nel tempo dal metodo diretto, il quale, ponendo l'accento sulla lingua parlata e abbandonando lo sforzo sterile e inadeguato di tradurre il vocabolo isolato, si indirizza alla comprensione unitaria del gruppo di parole e della frase intera, nella quale si esplica il senso e ogni termine assume la sua concreta funzione semantica. La scuola dimostra così di non essere estranea al progresso della linguistica, realizzando sul piano pratico l'insegnamento del de Saussure che «la langue est un système dont les thermes

1 V. R. Filipović Kontakti jezika u teoriji $i$ praksi (Lingue in contatto, teoria c prassi), Zagreb, 1971, Introduzione di P. Simleša, p. VIII. 
sont solidaires et où la valeur de l'un ne résulte que de la présence simultanée des autres». ${ }^{2}$

Già il grande grammatico e naturalista ceco Giovanni Komensky dimostrò nella sua Didactica maǵna che la metodica della lingue è direttamente legata alla scienza della logica e che la parola deve essere appresa in presenza dell'oggetto da essa simboleggiato. La didattica fa passi importanti specialmente con lo strutturalismo, nel cui solco si pone il metodo audiovisivo, per il principio deittico che lo informa e per lo sforzo di ristabilire il legame fra situazione reale rappresentata dalla figura e immagine acustica della lingua. Un importante contributo è rappresentato poi dalla così detta linguistica del contrasto, secondo la quale una delle difficoltà maggiori nell'apprendimento sta nell'interferenza fra la lingua prima e la ling'ua seconda, difficoltà che investe tutti i liveli, da quello fonetico, a quelle morfologico, sintattico, stilistico e socio-culturale. Il metodo della comparazione contrastiva nella preparazione dei manuali e nel processo dell'apprendimento è particolarmente efficace per superare le difficoltà, là dove le lingue si differenziano, e a correggere gli errori caratteristici commessi dai discenti.

Quale sviluppo e superamento dello strutturalismo, la nuova corrente della grammatica generativa trasformazionale propone oggi a sua volta una teoria linguistica e un sistema di regole che, determinando le strutture profonde e quelle superficiali della lingua, i loro rapporti e l'interpretazione semantica, dovrebbe permettere di riprodurre non solo testi appresi, ma di «produrre e riconoscere frasi che [...] non sono state mai usate precedentemente ${ }^{3}$

2. La lingua, come si sa, ha un aspetto razionale e un aspetto irrazionale ed è irrimediabilmente viziata dallo scompenso fra progresso materiale e relative possibilità espressive, le quali sono sempre in ritardo rispetto le necessità, ciò che spiega anche il trascinarsi dei fossili linguistici. Le lingue sono in compenso stimolate dalla tendenza a convergere, per rispondere al progressivo avvicinarsi delle culture nazionali, ciò che si manifesta attraverso i prestiti e i calchi linguistici; e infatti, facendo un confronto fra le lingue europee come sono parlate oggi e com'erano parlate tempo fa, si nota che esse si sono avvicinate sensibilmente in tutti i campi e specialmente in quello lessicale, per cui il Vendryes può notare che fra le lingue europee, specialmente fra quelle tipologicamente affini, la traduzione è abbastanza facile, potendosi spesso tradurre parola per parola. ${ }^{4}$

Il campo che oppone le difficoltà maggiori è quello della sintassi, particolarmente quello della fraseologia, difficoltà particolarmente sensibile per l'italiano, dato che questa lingua difetta di dizionari e raccolte

$2 \mathrm{~F}$. de Saussure, Cours de linguistique générale, Paris, 1955, p. 170.

3 G. C. Lepschy, La linguistica strutturale, Torino, 1966, p. 175.

4 V. G. Mounin, Les problèmes théoriques de la traduction, Paris, 1963, p. 217, dove riporta il pensiero di J. Vendryes e di altri linguisti. 
fraseologiche scientificamente impostate e spiegate, come per esempio, per il francese, il Dictionaire des locutions françaises del Rat. ${ }^{5}$

Il costrutto fraseologico, che è formato, come nota il Guiraud, da «une expression constituée par l'union de plusieurs mots formant une unité syntaxique et lexicologique $\ll{ }^{6}$ rampolla dai settori e per i motivi più diversi e più oscuri [usi e costumi, folclore, letteratura, etimologie popolari, eredità culturale, ecc.], e perciò ogni locuzione richiede generalmente una spiegazione a sé, che va ricercata nella storia, nella cultura, nella filologia, nell'interpretazione etimologica, e non sempre con sucesso, perché ogni locuzione è spesso un indovinello, di cui noi possediamo solo un elemento su dieci. E'perciò non solo inutile, ma quasi impietoso pretendere che lo straniero interpreti il senso racchiuso in una locuzione mai prima sentita, dato che la somma dei singoli elementi che la compongono non dà spesso il risultato che la logica si attenderebbe. Così, per esempio, il significato di una locuzione come «piantare baracca e burattini» deve essere necessariamente travisato dal traduttore croato che non conosca il suo valore metaforico; e infatti nella didascalia di un film è stata resa con la traduzione letterale 'osnivati kazalište lutaka», cosa che non vuol dire «mandare tutto al diavolo», ma «fondare il teatro delle marionette», frase che nel film è assolutamente priva di senso e lascia lo spettatore stupito e confuso. Prendiamo ancora una locuzione, del campo della politica, cioè «governo monocolore», diffusa recentemente dalla radio sotto la forma di «jednopartijska vlada», a sua volta tradotta e ritrasmessa in italiano come «governo unipartito», espressione che, se rende globalmente e con precisione il senso, se ci dà cioè il valore denotativo dell'originale, travisa l'aspetto connotativo che l'espressione «governo monocolore» sottende nella prassi politica italiana. In seguito i giornali hanno tradotto l'espressione con un calco perfetto, cioè «jednobojna vlada», dimostrando con ciò di aver afferrato interamente il problema espressivo, ma incorrendo nel pericolo di confondere il lettore comune, non abituato ad attribuire un colore ai partiti politici.

Non è nostra intenzione affrontare in questa sede il problema della traduzione delle locuzioni, ma ci limiteremo a notare che vi sono due repertori di locuzioni, quello nazionale, di uso interno, e quello internazionale, rappresentato da costrutti che hanno trovato fortuna oltre i confini etnici delle rispettive comunità nazionali e, veicolate dalla traduzione fedele come un calco 0 , più spesso, da una libera interpretazione lessicale e sintattica, concorrono efficacemente a creare un tessuto di nozioni comune alla nostra civiltà.

3. Benché la traduzione, come abbiamo visto, abbia avuto e abbia un'importanza sempre maggiore nella storia della civiltà, sia sorta prima di qualsiasi teoria linguistica e sia destinata a sopravviverle, molto si è

\footnotetext{
${ }_{5}$ M. Rat, Dictionnaire des locutions françaises, Paris, 1957, pp. 1-448.

6 P. Guiraud, Que sais-je?, Paris, 1962, p. 5.
} 


\section{Domenico Cernecca}

discusso e si discute in sede teorica se sia possibile tradurre. Gli studiosi si sono divisi in due schiere elaborando teorie adeguate e corrispondenti al grado di sviluppo della linguistica, di cui la traduzione è certamente parte non trascurabile, e all'angoln visuale dal quale il fatto linguistico è stato oservato.

Ambedue le opposte teorie, quella dei solipsisti e quella che crede nella possibilità della comunicazione interlinguistica, poggiano su principi ed osservazioni egualmente validi, ma partono da aspetti particolari della lingua. I primi infatti, movendo dalla non sistematicità del lessico e dalla peculiarità di ogni sintassi, giungono ad um disperato silenzio interlinguistico, non mai superabile, dato che, per citare esempi classici, l'idea dell'elefante di un indigeno del Kenia non troverà mai corrispondenza adeguata nella mente di un esquimese, né l'espressione "porte della città» potrà mai suscitare la visione corrispondente in un beduino adusato alla vita nomade e alla tenda piantata sulla sabbia dietro una duna o al riparo di una roccia; né si corrispondono appieno le idee dei colori nelle stesse lingue europee. Per questi studiosi l'ostacolo alla traduzione è in noi stessi, in quanto siamo prigionieri della nostra lingua materna la quale, come afferma il Whorf, è un vasto sistema di-strutture diverso da quello delle altre lingue, sistema nel quale si ordinano culturalmente le forme e le categorie per mezzo delle quali l'individuo non solo comunica, ma analizza la natura, rileva e trascura questi o quei gruppi di fenomeni e di relazioni in cui fluisce il suo modo di ragionare e per mezzo delle quali costruisce l'edificio della sua visione del mondo, ${ }^{7}$ $o$, più semplicemente, perché, come lapidariamente si esprime M. Cohen, ogni popolo ha la logica che rivela la sintassi della sua lingua materna. ${ }^{8}$ Né può, come nota $O$. Jerspersen, ${ }^{9}$ sperare l'adulto di ripetere il miracolo del bambino, il quale sente la lingua in tutte le situazioni possibili, e in tali condizioni in cui linguaggio e situazione reale corispondono esattamente l'uno all'altra e s'illustrano reciprocamente. La traduzione vera non è dunque possibile e si riduce, in fondo, a una interpretazione di ordine diverso, ma pur simile alla parafrasi degli antichi, i quali si accontentavano di riassumere l'argomento, più che preocuparsi di dare la corrispondenza essata della singola espressione.

Di fronte a queste ragioni nichiliste sta però la schiera degli ottimisti i quali, pur non potendo non tener conto degli argomenti dei negatori, si affidano all'esistenza degli universali linguistici e culturali, per cui certi concetti fondamentali sono pur tuttavia comuni a tutti gli uomini,

7 B. L. Whorf, Language thought and reality, New York, 1958, p. 214.

8 M. Cohen, Faits de linguistique et faits de pensée, Journal de Psychologie normale et pathologique, Paris, 1947, n. 4, p. 398.

${ }^{\circ} \mathrm{O}$. Jespersen, Language it nature depeloppement and origines, London, 1922 , p. 142 . 
e alla convergenza delle culture e perciò delle lingue, già a suo tempo rilevata dal Bally. ${ }^{10}$

Un argomento criticamente più convincente in favore della possibilità e della legittimità della traduzione è quello avanzato dal Mounin ${ }^{11}$ il quale, passando in rassegna e illustrando le varie teorie relative all'argomento, osserva che anche la traduzione va vista in prospettiva non statica, ma dinamica, per cui, essendo la visione del mondo e le lingue realtà non immobili, ma in continua evoluzione, ogni atto di traduzione è un contributo a una comprensione sempre più puntuale e feconda del dato linguistico straniero. Ciò vale non solo per le lingue affini, ma anche per lingue tipologicamente lontane l'una dall'altra, in quanto, anche se l'esperienza personale è incommensurabile, ed egualmente non sempre commisurabili sono, almeno in teoria, le unità di base di due lingue diverse, la comunicazione rimane tuttavia possibile, dato che il locutore e il traduttore partono da situazioni comuni. ${ }^{12}$

A questo punto non sarà forse inutile ricordare che nello stesso ordine d'idee si muove già Dante. Egli infatti, identificando, in termini naturalmente molto diversi e approssimativi, il concetto di prosa col lato denotativo e quello di poesia con quello connotativo del linguaggio, ci ha dato nel Convivio un'anticipazione illuminante sul problema della traduzione, notando che la prosa può passare da lingua a lingua, ma che «nulla cosa per legame musaico armonizzata [cioè la poesia] si può de la sua loquela transmutare sanza rompere tutta sua dolcezza ed armonia. E questa è la cagione per che Omero non si mutò di greco in latino come l'altre scritture che avemo da loro».13

In conclusione, il contrasto fra le due opposte correnti relative alla traduzione pare si sia sempre ridotto e si riduca tuttora alla possibilità di rendere il lato denotativo del fenomeno, che può essere esplicato dagli universali linguistici e dall'esperienza degli nomini sempre più ravvicinata e uniforme, mentre resiste ad ogni resa adeguata il lato connotativo che l'esegeta e il traduttore devono cercare di serrare il più da presso possibile, confidando che altri, dopo di loro, possa avvicinarsi più compiutamente alla sorgente dell'espressione. Ogni atto di traduzione è un concreto passo avanti verso traduzioni più fedeli, anche se non definitive.

${ }^{10}$ Ch. Bally, Traité de stylistique française, Heidelberg, 1909, vol. I, p. 48: «Les calques et les emprunts suffiraient à prouver l'existence de cette mentalité européenne».

${ }^{11} \mathrm{G}$. Mounin, Les problèmes théoriques de la traduction, Paris, 1963, p. 276.

12 G. Mounin, o.c., p. 278.

${ }^{13}$ D. Alighieri, Il Conoivio, Firenze, 1934, vol. I. p. 45. Nello stesso ordine di idee si pone, a distanza di secoli, il filosofo idealista Benedetto Croce il quale in Poesia, Bari 1943, a p, 100-101, nota che: «L'impossibilità della traduzione è la realtà stessa della poesia nella sua creazione e nella sua ricreazione», mentre la prosa, che sia veramente prosa e non prosa poetica è traducibile. 


\section{Sadržaj}

Autor promatra problem prevodenja sa tri aspekata. U prvom ilustrira značaj prevodenja i njegov razvitak kroz vjekove, u drugom zaustavlja se na frazeološkom obrtu kao specifičnom lingvističkom proizvodu svake nacije i odnosnim teškoćama pri prevođenju na tude jezike, dok u trećem raspravlja o samoj mogućnosti prevodenja konstatirajući da svaki konkretan prijevod predstavlja korak dalje prema što potpunijem shvačanju i pretakanju općeljudskog odnosno ličnog iskustva iz jezika u jezik. 